\title{
ON THE ONE-DIMENSIONAL BOLTZMANN EQUATION FOR GRANULAR FLOWS
}

\section{Dario Benedetto ${ }^{1}$ And Mario Pulvirenti ${ }^{1}$}

\begin{abstract}
We consider a Boltzmann equation for inelastic particles on the line and prove existence and uniqueness for the solutions.
\end{abstract}

Mathematics Subject Classification. 82C40, 76T25.

Received: February 19, 2001. Revised: June 19, 2001.

\section{INTRODUCTION}

The simplest models of granular flows are one-dimensional inelastic point particle systems (see e.g. [2] and references quoted therein). If a pair of particles with precollisional velocities $v$ and $v_{1}$ collides, the outgoing velocities $v^{\prime}$ and $v_{1}^{\prime}$ are defined in such a way as to dissipate kinetic energy, preserving the total momentum. In other words the collision law is:

$$
v^{\prime}=\frac{1}{2}\left(v+v_{1}\right)+\frac{1}{2}\left(v-v_{1}\right) h ; \quad v_{1}^{\prime}=\frac{1}{2}\left(v+v_{1}\right)-\frac{1}{2}\left(v-v_{1}\right) h
$$

where $h \in[0,1]$ is the one-dimensional restitution coefficient and measures the dissipation of the relative velocity $\left(v^{\prime}-v_{1}^{\prime}\right)=h\left(v-v_{1}\right)$.

Note that for $h=1$ the system becomes free while for $h=0$ we have the so-called sticky particle model: after a collision the particles proceed with the same velocity given by the momentum conservation.

From (1) we also deduce:

$$
v^{\prime}=v-\varepsilon\left(v-v_{1}\right) ; \quad v_{1}^{\prime}=v_{1}+\varepsilon\left(v-v_{1}\right)
$$

where $\varepsilon=\frac{1}{2}(1-h) \in[0,1 / 2]$ is another parameter measuring the degree of inelasticity of the collision.

Although $h$ (and hence $\varepsilon$ ) are usually assumed to be constant, one can also argue that, for some materials, when the relative velocity becomes very small, the collision is essentially elastic. Thus the restitution coefficient should be a suitable function of the relative velocity. In this paper, following [6] and references quoted therein, we assume the explicit phenomenological law:

$$
h\left(\left|v-v_{1}\right|\right)=\frac{1}{1+a\left|v-v_{1}\right|^{\gamma}}
$$

Keywords and phrases. Inelastic collisions, granular media, Boltzmann equation.

1 Dipartimento di Matematica, Università di Roma "La Sapienza", P.ale A. Moro 2, 00185 Rome, Italy.

e-mail: benedetto@mat.uniroma1.it, pulvirenti@axcasp.caspur.it 
where $a>0$ and $\gamma \in(0,1)$ are constants depending on the material. However all the considerations presented here can be easily extended to the case of any function $h$ of the relative velocity behaving like (3) for small $\left|v-v_{1}\right|$.

We anticipate that in the present paper we do not consider the case $\gamma=0$ (already considered in [3]).

In analogy with the standard (conservative) theory of rarefied gases, it is natural to derive a reduced description given in terms of a Boltzmann equation, which in our case reads as:

$$
\partial_{t} f+v \partial_{x} f=Q_{\gamma}(f, f)
$$

where

$$
Q_{\gamma}(f, f)(v)=\int \mathrm{d} v_{1}\left|v-v_{1}\right|\left(J f^{*} f_{1}^{*}-f f_{1}\right)
$$

Here $f=f(x, v, t)$ is the phase-space density function (as usual $f(x, v) \mathrm{d} x \mathrm{~d} v$ denotes the number of particles in the cell $[x, x+\mathrm{d} x] \times[v, v+\mathrm{d} v]$ in the phase space), $f, f_{1}, f^{*}$ and $f_{1}^{*}$ denote $f$ computed on the arguments $v, v_{1}, v^{*}, v_{1}^{*}$. Moreover, given the pair $\left(v, v_{1}\right),\left(v^{*}, v_{1}^{*}\right)$ denotes the incoming velocities:

$$
v^{*}=\frac{1}{2}\left(v+v_{1}\right)+\frac{1}{2 h^{*}}\left(v-v_{1}\right) ; \quad v_{1}^{*}=\frac{1}{2}\left(v+v_{1}\right)-\frac{1}{2 h^{*}}\left(v-v_{1}\right),
$$

where $h^{*}=h\left(\left|v^{*}-v_{1}^{*}\right|\right)$. Finally:

$$
J=\frac{1}{h^{*}\left(h^{*}+\left(v^{*}-v_{1}^{*}\right) h^{\prime} *\right.}=\frac{1}{h^{* 3}\left(1+a(1-\gamma)\left|v^{*}-v_{1}^{*}\right|^{\gamma}\right)},
$$

for which

$$
\mathrm{d} v \mathrm{~d} v_{1} J\left|v-v_{1}\right|=\mathrm{d} v^{*} \mathrm{~d} v_{1}^{*}\left|v^{*}-v_{1}^{*}\right|
$$

In this paper we study the Cauchy problem for equation 4 and present a global existence theorem. The nonhomogeneous problem is considerably more difficult than the homogeneous one with regards to the standard kinetic theory. The main difficulty, when an energy dissipation is present, is related to the absence of an $a$ priori entropy control, due to the fact that the system has the tendency to cluster. In the present paper we shall consider only the case $\gamma>0$ and this makes the clustering effect less effective. For the case $\gamma=0$, considered in [3], we have only partial results and the problem of giving a global existence result (or evidence of collapses or blow-up) is still open for general initial conditions.

\section{Formal PROPERTIES OF THE SOLUTION}

In this section we deduce some properties of the solutions (if any) to equation 4 , which will be crucial in the construction of the solution to the Cauchy problem. The main point is the control of the entropy of the system which, in our dissipative context, is not trivial. Then, following a well known classical argument, we can derive an uniform absolute continuity bound which allows us to pass from a short time to a globally in time result. We only sketch this last part in Section 4 for sake of completeness.

We underline once more that the entropy inequality we are able to obtain holds only for $\gamma>0$.

We observe preliminary that:

$$
\int \mathrm{d} v \phi(v) Q_{\gamma}(f, f)(v)=\frac{1}{2} \iint \mathrm{d} v \mathrm{~d} v_{1}\left|v-v_{1}\right|\left\{\phi\left(v^{\prime}\right)+\phi\left(v_{1}^{\prime}\right)-\phi(v)-\phi\left(v_{1}\right)\right\} f(x, v, t) f\left(x, v_{1}, t\right) .
$$

Note that if $\phi$ is convex, then $\left\{\phi\left(v^{\prime}\right)+\phi\left(v_{1}^{\prime}\right)-\phi(v)-\phi\left(v_{1}\right)\right\}$ in (8) is negative so that:

$$
\frac{\mathrm{d}}{\mathrm{d} t} \int \mathrm{d} v \int \mathrm{d} x \phi(v) f(x, v, t) \leq 0
$$


and this expresses the tendency of the system to cluster. In particular the total kinetic energy is decreasing in time.

In constructing the solution it is crucial a control of the Entropy functional (which can increase in our context) to avoid concentration.

\section{Entropy inequality}

Defining

$$
H(t)=\int \mathrm{d} x \int \mathrm{d} v f \log f(x, v, t)
$$

we have

$$
\dot{H}(t) \leq \frac{1}{2} \int \mathrm{d} x \int \mathrm{d} v \int \mathrm{d} v_{1}(J-1)\left|v-v_{1}\right| f(x, v, t) f\left(x, v_{1}, t\right) .
$$

The proof of (11) is consequence of a direct computation using (8) and the inequality:

$$
(\log h-\log k) k \leq \frac{k}{\xi h+(1-\xi) k}(h-k) \leq(h-k)
$$

which is valid for any $h, k \geq 0$ and some $\xi \in(0,1)$.

Using the explicit form of $J$,

$$
J-1=a\left|w^{*}\right|^{\gamma} \frac{2+\gamma+3 a\left|w^{*}\right|^{\gamma}+a^{2}\left|w^{*}\right|^{2 \gamma}}{1+a(1-\gamma)\left|w^{*}\right|^{\gamma}} \leq c\left(\left|w^{*}\right|^{\gamma}+\left|w^{*}\right|^{2 \gamma}\right),
$$

where $w^{*}=v^{*}-v_{1}^{*}$ and $c$ is a constant depending on $a$ and $\gamma$. Being $\left|w^{*}\right| \leq|w|\left(1+a|w|^{\gamma}\right)$ we obtain, as corollary of inequality (11),

$$
\dot{H}(t) \leq C \int \mathrm{d} x \int \mathrm{d} v \int \mathrm{d} v_{1}\left(\left|v-v_{1}\right|^{1+\gamma}+\left|v-v_{1}\right|^{1+4 \gamma}\right) f(x, v, t) f\left(x, v_{1}, t\right),
$$

where $C$ is a constant depending on $a$ and $\gamma$.

Therefore to control $H$ we must try and control the quantity

$$
\mathcal{I}_{\alpha}(T)=\int_{0}^{T} \mathrm{~d} t \int \mathrm{d} x \int \mathrm{d} v \int \mathrm{d} v_{1}\left|v-v_{1}\right|^{1+\alpha} f(x, v, t) f\left(x, v_{1}, t\right)
$$

for $\alpha \in(0,1]$.

We estimate $\mathcal{I}_{\alpha}$ by extending an argument due to Bony [4].

Define, for any odd function $\phi$ :

$$
I_{\phi}(t)=\int \mathrm{d} x \int \mathrm{d} y \int \mathrm{d} v \int \mathrm{d} w \mathcal{X}(x<y) \phi(v-w) f(x, v, t) f(y, w, t)
$$

where $\mathcal{X}$ is the characteristic function. Then

$$
\begin{aligned}
\dot{I}_{\phi}(t)= & -\int \mathrm{d} x \int \mathrm{d} v \int \mathrm{d} w \phi(v-w)(v-w) f(x, v, t) f(x, w, t) \\
+ & \frac{1}{2} \int \mathrm{d} x \int \mathrm{d} y \int \mathrm{d} v \int \mathrm{d} v_{1} \int \mathrm{d} w\left|v-v_{1}\right|(\mathcal{X}(x<y)-\mathcal{X}(y<x)) f(y, w, t) \\
& f(x, v, t) f\left(x, v_{1}, t\right)\left[\phi\left(v^{\prime}-w\right)+\phi\left(v_{1}^{\prime}-w\right)-\phi(v-w)-\phi\left(v_{1}-w\right)\right] .
\end{aligned}
$$

We shall use this identity for $\phi_{\alpha}=\operatorname{sgn}(v)|v|^{\alpha}$. 
Integrating with respect to time, we have:

$$
\begin{aligned}
\mathcal{I}_{\alpha}(T)= & I_{\phi_{\alpha}}(0)-I_{\phi_{\alpha}}(T) \\
+ & \frac{1}{2} \int_{0}^{T} \int \mathrm{d} x \int \mathrm{d} y \int \mathrm{d} v \int \mathrm{d} v_{1} \int \mathrm{d} w\left|v-v_{1}\right|(\mathcal{X}(x<y)-\mathcal{X}(y<x)) f(y, w, t) \\
& f(x, v, t) f\left(x, v_{1}, t\right)\left[\phi_{\alpha}\left(v^{\prime}-w\right)+\phi_{\alpha}\left(v_{1}^{\prime}-w\right)-\phi_{\alpha}(v-w)-\phi_{\alpha}\left(v_{1}-w\right)\right] .
\end{aligned}
$$

Note that, if $\alpha=1$, using the momentum conservation, the last term in the right hand side of equation (18) vanishes and we have (see [4]):

$$
\mathcal{I}_{1}(T)=I_{\phi_{1}}(0)-I_{\phi_{1}}(T)
$$

For $\alpha<1$ we need to estimate the last term in the right half side of equation (18). In doing this we arrive to an inequality (see Eq. (24) below) which involves $\mathcal{I}_{\alpha^{\prime}}$ with $\alpha^{\prime}>\alpha$. The idea is to iterate this relation to arrive to $\alpha=1$ and hence to estimate $\mathcal{I}_{\alpha}$ in term of $I_{\phi_{\alpha}}$.

Namely for $\alpha<1$, assuming $v^{\prime}>v$ (the other case $v^{\prime} \leq v$ can be handled analogously), we have:

$$
\begin{aligned}
\int \mathrm{d} y \int \mathrm{d} w f(y, w, t)\left[\phi_{\alpha}\left(v^{\prime}-w\right)-\phi_{\alpha}(v-w)\right]= & \int \mathrm{d} y \int_{v}^{v^{\prime}} \mathrm{d} w f(y, w, t)[\ldots]+\int \mathrm{d} y \int_{-\infty}^{v} \mathrm{~d} w f(y, w, t)[\ldots] \\
& +\int \mathrm{d} y \int_{v^{\prime}}^{+\infty} \mathrm{d} w f(y, w, t)[\ldots]
\end{aligned}
$$

Moreover, if $w \in\left(v, v^{\prime}\right)$ :

$$
[\ldots]=\left|v^{\prime}-w\right|^{\alpha}+|v-w|^{\alpha} \leq 2\left|v^{\prime}-v\right|^{\alpha}=2 \varepsilon^{\alpha}\left|v-v_{1}\right|^{\alpha} \leq 2^{1-\alpha} a^{\alpha}\left|v-v_{1}\right|^{(1+\gamma) \alpha}
$$

If $w<v$ :

$$
\begin{aligned}
{[\ldots] } & =\left|v^{\prime}-w\right|^{\alpha}-|v-w|^{\alpha}=\left|v-w-\varepsilon\left(v-v_{1}\right)\right|^{\alpha}-|v-w|^{\alpha} \\
& =\varepsilon^{\alpha}\left|v-v_{1}\right|^{\alpha}\left(\left|\frac{v-w}{\varepsilon\left(v-v_{1}\right)}-1\right|^{\alpha}-\left|\frac{v-w}{\varepsilon\left(v-v_{1}\right)}\right|^{\alpha}\right) \leq M 2^{-\alpha} a^{\alpha}\left|v-v_{1}\right|^{(1+\gamma) \alpha}
\end{aligned}
$$

where $M=\max _{\xi \in \mathbb{R}}\left(|\xi-1|^{\alpha}-|\xi|^{\alpha}\right)$. The term $w>v^{\prime}$ can be treated in the same way.

In conclusion:

$$
\int \mathrm{d} y \int \mathrm{d} w f(y, w, t)\left[\phi_{\alpha}\left(v^{\prime}-w\right)-\phi_{\alpha}(v-w)\right] \leq C m\left|v-v_{1}\right|^{(1+\gamma) \alpha}
$$

where $C$ is a constant and $m=\int \mathrm{d} x \int \mathrm{d} v f(t)$ is the total mass of the system.

Inserting this estimate in the right hand side of equation (18) we readily arrive to the inequality:

$$
\mathcal{I}_{\alpha}(T) \leq I_{\phi_{\alpha}}(0)-I_{\phi_{\alpha}}(T)+C m \mathcal{I}_{(1+\gamma) \alpha}(T)
$$

from which:

$$
\mathcal{I}_{\alpha}(T) \leq \sum_{k=0}^{n-1}(C m)^{k}\left(I_{\phi_{(1+\gamma)^{k}}}(0)-I_{\phi_{(1+\gamma)^{k}}}(T)+(C m)^{n} \mathcal{I}_{(1+\gamma)^{n} \alpha}\right.
$$

Setting $\alpha_{n}=\frac{1}{(1+\gamma)^{n}}$, using (19) we obtain:

$$
\mathcal{I}_{\alpha_{n}}(T) \leq \sum_{k=0}^{n}(C m)^{k}\left(I_{\phi_{(1+\gamma)^{k-n}}}(0)-I_{\phi_{(1+\gamma)^{k-n}}}(T)\right)
$$


Note now that

$$
\left|I_{\alpha_{n}}(T)\right| \leq C\left(\int \mathrm{d} x \int \mathrm{d} v|v|^{\alpha} f(x, v, t)\right)^{2} \leq C\left(\int \mathrm{d} x \int \mathrm{d} v\left(1+|v|^{2}\right) f(x, v, t)\right)^{2}
$$

and hence:

$$
\mathcal{I}_{\alpha_{n}}(T) \leq C(m, E)
$$

where $C(m, E)$ is a constant depending only on the mass $m$ and the energy $E=\frac{1}{2} \int \mathrm{d} x \int \mathrm{d} v v^{2} f_{0}(x, v)$ of the initial datum $f_{0}$.

Finally, for any $\alpha \in(0,1]$ we can find $n$ such that $\alpha \in\left[\alpha_{n+1}, \alpha_{n}\right]$. Since

$$
\mathcal{I}_{\alpha} \leq \mathcal{I}_{\alpha_{n}}+\mathcal{I}_{\alpha_{n+1}}
$$

we conclude that:

$$
\mathcal{I}_{\alpha}(T) \leq C(m, E) .
$$

Another property of the solution we are going to use is the following. Suppose that $v^{2}>v_{1}^{2}$. Then

$$
{v^{\prime}}^{2}=v^{2}\left[(1-2 \varepsilon)+\varepsilon^{2}\right]+\varepsilon^{2} v_{1}+\left(2 \varepsilon-2 \varepsilon^{2}\right) v v_{1} \leq v^{2}\left[(1-2 \varepsilon)+\varepsilon^{2}\right]+\left(2 \varepsilon-2 \varepsilon^{2}\right) v^{2} \leq v^{2}
$$

therefore the collision mechanism cannot increase the largest velocity. Hence if

$$
f_{0}(x, v)=0 \quad \text { when } \quad|v|>V
$$

for a given $V>0$ then:

$$
f(x, v, t)=0 \text { when } \quad|v|>V \text { for } t>0 .
$$

As consequence, under condition (31) we have, for $\alpha>\beta$ :

$$
\mathcal{I}_{\alpha}(T) \leq\left(1+V^{\alpha-\beta}\right) \mathcal{I}_{\beta}(T)
$$

and by (14) and (29) we can control the entropy production. In conclusion:

Proposition 1. Suppose $f=f(x, v, t)$ be a classical solution to equation (4) with initial datum $f_{0}$ satisfying (31) and such that $H\left(f_{0}\right)=\int \mathrm{d} x \int \mathrm{d} v f_{0} \log f_{0}<+\infty$. Then there exist a constant $C$ depending on $m, E$ and $H\left(f_{0}\right)$ for which $H(t) \equiv H(f(t)) \leq C$.

\section{The Cauchy problem for small Data}

We shall now sketch a global existence and uniqueness result for the solution of equation (4) for small values of $\int \mathrm{d} x \mathrm{~d} v f_{0}(x, v)$ (see [1] and [3]). In the next section we will use this result and the a priori estimate for the entropy production, in order to obtain the global existence and uniqueness theory for all initial data.

Theorem 1. Let $f_{0} \in \mathbf{L}_{\infty}(\mathbb{R}), f_{0} \geq 0$. Then, if the total mass $\left\|f_{0}\right\|_{1}$ is small, there exists an unique mild, bounded solution in $\mathbf{L}_{\infty}$ of (4) with initial datum given by $f_{0}$.

Proof. Let $f^{\#}(x, v, t)=f(x+v t, v, t)$. It is easy to realize that $f^{\#}$ satisfies

$$
f^{\#}(x, v, t) \leq f_{0}(x, v)+\int_{0}^{t} \mathrm{~d} s \int \mathrm{d} v_{1}\left|v-v_{1}\right| J f^{\#}\left(x+\left(v-v^{*}\right) s, v^{*}, s\right) f^{\#}\left(x+\left(v-v_{1}^{*}\right) s, v_{1}^{*}, s\right) .
$$

Let

$$
F(x, v)=\sup _{t \geq 0} f^{\#}(x, v, t)
$$


From (34):

$$
\|F\|_{1} \leq\left\|f_{0}\right\|_{1}+\int_{0}^{\infty} \mathrm{d} s \int \mathrm{d} x \mathrm{~d} v \mathrm{~d} v_{1}\left|v-v_{1}\right| J F\left(x+\left(v-v^{*}\right) s, v^{*}\right) F\left(x+\left(v-v_{1}^{*}\right) s, v_{1}^{*}\right) .
$$

Coming back to the precollisional variables of integration, using (7) we have that:

$$
\|F\|_{1} \leq\left\|f_{0}\right\|_{1}+\int_{0}^{\infty} \mathrm{d} s \int \mathrm{d} x \mathrm{~d} v \mathrm{~d} v_{1}\left|v-v_{1}\right| F\left(x+\left(v^{\prime}-v\right) s, v\right) F\left(x+\left(v^{\prime}-v_{1}\right) s, v_{1}\right) .
$$

Putting $y=x+\left(v^{\prime}-v\right) s$, and $z=x+\left(v^{\prime}-v_{1}\right) s$, by elementary computations we obtain:

$$
\mathrm{d} x \mathrm{~d} s\left|v-v_{1}\right|=\mathrm{d} y \mathrm{~d} z
$$

then

$$
\|F\|_{1} \leq\left\|f_{0}\right\|_{1}+\|F\|_{1}^{2}
$$

and therefore

$$
F \leq \frac{1}{2}\left(1-\sqrt{1-4\left\|f_{0}\right\|_{1}}\right)
$$

if

$$
\left\|f_{0}\right\|_{1}<\frac{1}{4}
$$

Now we are able to prove an $\mathbf{L}_{\infty}$ a priori estimate on $f(x, v, t)$.

Let

$$
G=\sup _{x, v, t \geq 0} f^{\#}(x, v, t)
$$

From (34):

$$
G \leq\left\|f_{0}\right\|_{\infty}+G \int_{0}^{\infty} \mathrm{d} s \int \mathrm{d} v_{1}\left|v-v_{1}\right| J f^{\#}\left(x+\left(v^{*}-v_{1}^{*}\right) s, v_{1}^{*}, s\right) .
$$

We change the variables $\left(s, v_{1}\right) \rightarrow\left(y, v_{1}^{*}\right)$, with $y=x+\left(v-v_{1}^{*}\right) s$.

Taking into account that

$$
\frac{\partial v_{1}^{*}}{\partial v_{1}}=\frac{2+a(3-\gamma)\left|w^{*}\right|^{\gamma}+a^{2}(1-\gamma)\left|w^{*}\right|^{2 \gamma}}{2\left(1+a(1-\gamma)\left|w^{*}\right|^{\gamma}\right)}
$$

and that $\left|v-v_{1}^{*}\right|=\frac{1+h\left(\left|w^{*}\right|\right)}{2}\left|w^{*}\right|$, we obtain

$$
J\left|v-v_{1}\right| \mathrm{d} s \mathrm{~d} v_{1}=\frac{2 J h\left(\left|w^{*}\right|\right)}{1+h\left(\left|w^{*}\right|\right)} \frac{\partial v_{1}}{\partial v_{1}^{*}} \mathrm{~d} y \mathrm{~d} v_{1}^{*}=4 \frac{(1+z)^{2}}{2+z} \frac{1}{2+(3-\gamma) z+(1-\gamma) z^{2}} \mathrm{~d} y \mathrm{~d} v_{1}^{*},
$$

where $z=a\left|w^{*}\right|^{\gamma}$. By an explicit estimation of this expression, from (42) we conclude that

$$
G \leq\left\|f_{0}\right\|_{\infty}+2\|F\|_{1} G
$$

Therefore $G$ is bounded if

$$
\|F\|_{1}<\frac{1}{2}
$$

With this a priori bound the construction of the solutions is standard. 


\section{The Cauchy Problem for arbitrary Data}

Theorem 4.1. Let $f_{0} \in \mathbf{L}_{\infty}(\mathbb{R}), f_{0} \geq 0, f_{0}(x, v)=0$ if $|v| \geq V$. Then there exists an unique mild, bounded solution in $\mathbf{L}_{\infty}$ of (4) with initial datum given by $f_{0}$.

The proof of Theorem 4.1 follows from Theorem 3.1 by using the entropy bound in Proposition 1, and an argument due to Tartar [5] which we repeat here for completeness.

Proof. Being $f(x, v, t)=0$ for $|v|>V$ and any time, the solution at time $\bar{t}$ in $\bar{x}$ depends only on $f_{0}(x, v)$ restricted to the interval $x \in(\bar{x}-V \bar{t}, \bar{x}+V \bar{t})$. We can choose $\bar{t}$ such that

$$
\sup _{\bar{x}} \int_{\bar{x}-V \bar{t}}^{\bar{x}+V \bar{t}} \mathrm{~d} x \int \mathrm{d} v f_{0}(x, v)
$$

is sufficiently small in order to verify the inequalities (40), (41) and (43). This gives a small time existence result for all initial data.

The entropy estimate in Proposition 1 allows us to choose $\bar{t}$ independently from $f_{0}$. Namely, let $I$ be an interval of $\mathbb{R}$, with meas $I=|I|<1$ :

$$
\begin{aligned}
\int_{I} \mathrm{~d} x \int \mathrm{d} v f(x, v, t) & =\int \mathrm{d} v \int_{I \cap\left\{f \leq|I|^{-\frac{1}{2}}\right\}} \mathrm{d} x f(x, v, t)+\int \mathrm{d} v \int_{I \cap\left\{f>|I|^{-\frac{1}{2}}\right\}} \mathrm{d} x f(x, v, t) \\
& \leq 2 V|I|^{\frac{1}{2}}+\frac{2}{|\log | I||} \int \mathrm{d} x \mathrm{~d} v f(x, v, t)|\log f(x, v, t)| .
\end{aligned}
$$

We conclude the proof by noticing that the last estimate depends only on the entropy and the total mass, and then the proof of the local in time existence can be iterated to reach any time.

\section{REFERENCES}

[1] L. Arkeryd, Existence theorems for certain kinetic equations and large data. Arch. Rational Mech. Anal. 103 (1988) $139-149$.

[2] D. Benedetto, E. Caglioti and M. Pulvirenti, A kinetic equation for one-dimensional granular media. RAIRO Modél. Math. Anal. Numér. 31 (1997) 615-641.

[3] D. Benedetto, E. Caglioti and M. Pulvirenti, A one-dimensional Boltzmann equation with inelastic collisions. Rend. Sem. Mat. Fis. Milano LXVII (1997) 169-179.

[4] J.-M. Bony, Solutions globales bornées pour les modèles discrets de l'équation de Boltzmann en dimension 1 d'espace, in Actes Journées Équ. Dériv. Part. 16, St.-Jean-de-Monts (1987).

[5] L. Tartar, Existence globale pour un système hyperbolique semi-linéaire de la théorie cinétique des gaz, in Séminaire GoulaouicSchwartz 1975-76, Équat. Dériv. Part. Anal. Fonct., Exposé I, École Polytechnique, Palaiseau (1976).

[6] G. Toscani, One-dimensional kinetic models of granular flows. Math. Model. Numer. Anal. 34 (2000) 1277-1291.

To access this journal online: www.edpsciences.org 